\title{
Games Played in a Contracting Environment*
}

\author{
V. Bhaskar \\ Department of Economics \\ University College London \\ Gower Street \\ London WC1 6BT
}

February 2008

\begin{abstract}
We analyze normal form games where a player has to pay a price to a supplier in order to play a specific action. Our focus is on supplier competition, arising from the fact that distinct suppliers supply different players, and possibly the different actions of the same player. With private contracts, where a player only observes the prices quoted by his own suppliers, the set of equilibrium distribution over player actions coincides with the set of equilibrium distribution when all actions are supplied competitively, at cost. With public contracts, the two distributions differ dramatically even in simple games.

Keywords: multi-party contracting, mixed strategy equilibria, marginal contribution, perfect substitutes, perfect complements.

JEL Classification Nos: C73, L13, L14.
\end{abstract}

${ }^{*}$ I am grateful to Larry Samuelson, Ilya Segal, an anonymous referee and an associate editor for very helpful comments. 


\section{Introduction}

This paper analyzes normal form games where a player has to pay a price to a supplier in order to play a specific action. Our focus is on supplier competition, arising from distinct suppliers supplying different players, and possibly the different actions of the same player. In the extensive form game, suppliers simultaneously announce prices. Players then choose actions, and their payoffs equal to the original payoffs minus the price paid for any action taken. The payoff of a supplier who makes a sale equals the price received minus the cost of provision. We study two different informational scenarios. In the game with private contracts, each player only observes the profile of prices that are applicable for his own actions. In the game with public contracts, players observe all the prices set by all suppliers. Our focus is on the interplay between supplier pricing decisions and strategic interaction between players in the normal form game.

In the case of private contracts, we provide a complete characterization of equilibrium outcomes. The set of equilibrium distributions over player action profiles coincides with the set of equilibrium distributions when all inputs are supplied competitively, at cost. Furthermore, in any equilibrium, a supplier earns his marginal contribution to a player's payoff at the action profile played.

With public contracts our results are very different, since supplier competition has subtle and complex effects. Even in simple games like the prisoners' dilemma, public contracts may result in very different outcomes, where the players randomize across different actions. These phenomena arise since a supplier to a player may be able to influence the player's opponent's behavior by the choice of price, thereby affecting the player's payoff, and the amount that he is willing to pay. Our second insight concerns mixed equilibria competing suppliers for the same player may act partially as though they are producing complementary goods, and in part as though they are producing substitutes, whereas under private contracting, they compete intensely since they produce perfect substitutes.

\begin{tabular}{|l|l|l|}
\hline & $C$ & $D$ \\
\hline$C$ & 1,1 & $-\ell_{1}, 1+g_{2}$ \\
\hline$D$ & $1+g_{1},-\ell_{2}$ & 0,0 \\
\hline
\end{tabular}

Fig. 1: Prisoner's Dilemma 
To illustrate our arguments, consider the simple prisoner's dilemma game set out in Fig.1. Each player $i \in\{1,2\}$ may choose action $C$ unilaterally, without contracting with any supplier. However, if he chooses to play action $D$ he must contract with a monopoly supplier, where player 1's supplier differs from player 2's. We present two stylized economic examples which correspond to this game.

1. Two firms are located in different countries and engaged in Cournot competition in a single market. Each firm initially has constant marginal costs, and may purchase equipment from a local supplier (action $D)$ in order to reduce its marginal costs. Purchase decisions are public information, and the gross payoffs from these decisions are Cournot equilibrium profits, minus the cost of production of the equipment this is assumed to be sufficiently low in both countries, giving rise to a prisoner's dilemma game. With linear demand, the equipment purchase decisions are strategic substitutes: $g_{i}>\ell_{i}>0$ for both firms.

2. Two firms are in distinct territories, each containing a unit measure of consumers. The firms offer products of differing quality, and each may advertise in its rival's territory (action $D$ ), buying advertising services from a local monopolist. In the absence of advertising, each firm serves consumers in its own territory. If firm $i$ advertises and firm $j$ does not, then firm $i$ gets a fraction $\lambda$ of the other firm's consumers, so that the market sizes are $(1+\lambda)$ and $(1-\lambda)$ respectively. If both firms advertise, a consumer in a firm $i$ 's own territory may also see $i$ 's advertisement with probability $\frac{\lambda}{k}, k>1$. A consumer who sees both advertisements chooses the high quality firm (firm 2), giving the first firm $1-\frac{\lambda^{2}}{k}$ consumers and the second $1+\frac{\lambda^{2}}{k}$ consumers. The cost of production of advertising is negligible so that gross payoffs equal sales. After renormalizing payoffs, this gives rise to a prisoner's dilemma as in Fig. 1, with $g_{1}>\ell_{1}>0$ and $0<g_{2}<\ell_{2}$.

If the prisoner's dilemma is played with private contracts, there is a unique equilibrium where $(D, D)$ is played, and each supplier earns $\ell_{i}$, his marginal contribution to the player's payoff at this action profile. With public contracts, we have the same equilibrium outcomes as under private contracts as long as a) $g_{i}-\ell_{i}$ is positive for both players, i.e. the defect decisions are strategic substitutes for both, or b) $g_{i}-\ell_{i}$ is negative for both players, 
i.e. the defect decisions are strategic complements for both players. However, if $g_{1}>\ell_{1}$ and $\ell_{2}>g_{2}$, there is no equilibrium where $(D, D)$ is played with probability one. Intuitively, if supplier 2 prices at $\ell_{2}$ and extracts his marginal contribution, then supplier 1 has an incentive to increase his price above $\ell_{1}$ - in any equilibrium of the resulting subgame, player 1 must play $D$ with probability one. Interestingly, it is the supplier of the weaker firm the firm that that produces low quality - that tries to manipulate its rival's behavior. Consequently, there is no pure strategy equilibrium; equilibrium prices are random, resulting in a correlated distribution over action profiles.

This paper is related to the literature on strategic delegation (Fershtman and Judd [5], Sklivas [18], Vickers [19], Fershtman et. al. [6]), which emphasizes that delegation with public contracts allowed a principal to secure favorable outcomes. Katz [11], Fershtman and Kalai [7] and Kockesen and Ok [12] examine the implications of private contracting in this context, while Caillaud et. al. [4] allow public contracting followed by private renegotiation. In the strategic delegation context, an agent requires to contract with the principal to take any action in the game, the principal effectively has monopoly power over all the actions. In contrast, we have focus on the situation where any supplier has limited monopoly power, over a subset of player actions, and thus supplier competition plays an important role. Our paper is also an instance of multi-party contracting, and the literature in this area includes common agency models (Bernheim and Whinston, [2]), and vertical contracting between a single principal and many agents (Hart and Tirole, [9], McAfee and Schwartz, [13], and Segal [15]). Its connections are closest to the case where there are many agents as well as principals, as in Prat and Rustichini, [14], or Jackson and Wilkie [10], where players in the game may make side payments to other players). However, while these papers allow general forms of contracting between principals and agents, and focus on the question of whether contracting ensures efficiency, a "principal" in our context has limited power, since he may only demand a transfer in the event that the player chooses an action the principal controls. The actions taken by "agents" in our model have no direct payoff consequences for principals, unlike common agency models.

The rest of this paper is as follows. Section 2 sets out the basic model of a game played in a contracting environment. Section 3 analyzes private contracting, while section 4 focuses on public contracts. For the sake of smooth exposition, all proofs are in the appendix. 


\section{The Model}

We now define a game played in a contracting environment. We use the term player for someone who plays the game in question, and the term supplier to denote someone with whom a player may need to contract in order to adopt some action in the game. Let $I$ denote the finite set of players and let each $i \in I$ have a finite action set $A_{i}$, whose generic element will be denoted by $a_{i}^{j}$ or $a_{i}$. Let $A=\times_{i \in I} A_{i}$ be the set of action profiles, and let $g_{i}: A \rightarrow R$ be the gross payoff of player $i$. These gross payoffs at the profile $\mathbf{a}=\left(a_{i}\right)_{i \in I}$ will in general differ from the usual (net) payoffs of a player since she may have to contract with a supplier in order to be able to play the action $a_{i}$. Let $\bar{A}_{i} \subset A_{i}$ be the set of actions for which the player needs a supplier. For simplicity, we shall assume that for any player $i$ and any action $a_{i}^{j} \in \bar{A}_{i}$, there exists exactly one supplier, $\lambda\left(a_{i}^{j}\right) . \Lambda_{i}=\left\{\lambda\left(a_{i}^{j}\right)\right\}_{a_{i}^{j} \in \bar{A}_{i}}$ denotes the set of suppliers for player $i$. Let $p_{i}^{j}$ denote the price which is charged by supplier $\lambda\left(a_{i}^{j}\right)$ for enabling the action $a_{i}^{j}$, and let $\mathbf{p}_{i}=\left(p_{i}^{j}\right)_{a_{i}^{j} \in \bar{A}_{i}}$. The price is contingent only on whether or not the action $a_{i}^{j}$ is played, since only this is verifiable. We assume it cannot be made contingent upon the action profile played, since this is not verifiable. If $a_{i}^{j} \notin \bar{A}_{i}$ we set the price of this action, $p_{i}^{j}$, to zero. The net payoff at the profile $\mathbf{a}=\left(a_{i}^{j}, a_{-i}\right)$ where $a_{-i}$ is the vector of actions of players $h \neq i$, is given by

$$
u_{i}\left(a_{i}^{j}, a_{-i}, \mathbf{p}_{i}\right)=g_{i}\left(a_{i}^{j}, a_{-i}\right)-p_{i}^{j} .
$$

If player $i$ plays action $a_{i}^{j} \in \bar{A}_{i}$, the payoff to supplier $\lambda\left(a_{i}^{j}\right)$ is given by $p_{i}^{j}-c_{i}^{j}$, where $c_{i}^{j}$ is the cost of supplying this action. If the player does not play action $a_{i}^{j}$, the payoff to the supplier of this action is zero. Let us normalize prices and gross payoffs by measuring them net of cost, so that a zero price corresponds to pricing at cost. Henceforth, the gross payoff $g_{i}\left(a_{i}, a_{-i}\right)$ will denote the payoff when player pays the cost of action $a_{i}$. We extend, in the usual way, the gross payoff function $g_{i}$ to mixed action profiles: $g_{i}\left(\alpha_{i}, \alpha_{-i}\right)$ is the payoff to $\alpha_{i} \in \Delta\left(A_{i}\right)$ when $\alpha_{-i} \in \times_{h \neq i} \Delta\left(A_{h}\right)$ is the vector of (mixed) actions played by the other players.

We assume that each supplier is a monopolist in the market for the action that he enables - this is easily relaxed. Our other assumptions are as follows.

Assumption A1. (No Complementary Inputs): For any player $i$ and any action $a_{i}^{j}$, no more than one supplier is required. 
Assumption A2. For every player $i$ there exists an action $a_{i}^{0}$ such that no input is required to play this action.

This assumption ensures that the minimum payoff that any player in $I$ can receive is bounded and given by $\min _{a_{-i}} g_{i}\left(a_{i}^{0}, a_{-i}\right)$. In the public contracts case, this assumption is necessary in order to ensure existence of equilibrium, since otherwise, competing suppliers may act as though they are producing complementary goods. In the private contracts case, this ensures a finite "choke price", in the absence of which there may be mixed pricing equilibria with an unbounded support, as in [1].

Assumption A3. A supplier supplies at most one player, i.e. $\Lambda_{i}$ and $\Lambda_{j}$ are disjoint if $i \neq j$.

This assumption plays an essential role in our analysis of private contracts, as we shall explain shortly.

A point of reference is the normal form game $G=\langle I, A, g\rangle$, where all actions are supplied at cost, and players' net payoffs equal their gross payoffs. The payoffs we write down, in the various examples, will be of the game $G$. Let $E^{G}$ denote the set of Nash equilibria of $G$.

Let $\boldsymbol{\alpha}=\left(\alpha_{i}, \alpha_{-i}\right) \in E^{G}$, and let $a_{i}^{j} \in \bar{A}_{i}$ belong to the support of $\alpha_{i}$. The marginal contribution of supplier $\lambda\left(a_{i}^{j}\right)$ at the profile $\alpha$ is denoted by $\delta\left(a_{i}^{j}, \boldsymbol{\alpha}\right)$, and equals the gross payoff loss that player $i$ suffers from playing her best alternative action $a_{i}^{k}$ from a different supplier, i.e. where $a_{i}^{k}$ is such that $\lambda\left(a_{i}^{k}\right) \neq \lambda\left(a_{i}^{j}\right)$. That is, $\delta\left(a_{i}^{j}, \alpha\right)=g_{i}(\alpha)-\max _{a_{i}^{k}: \lambda\left(a_{i}^{k}\right) \neq \lambda\left(a_{i}^{j}\right)} g_{i}\left(a_{i}^{k}, \alpha_{-i}\right)$. If $\alpha_{i}$ assigns probability only to actions supplied by $\lambda\left(a_{i}^{j}\right)$, then $\delta\left(a_{i}^{j}, \alpha\right)$ is the payoff loss suffered by $i$ when he chooses the next best action that is not supplied by $\lambda\left(a_{i}^{j}\right)$. If $\alpha_{i}$ assigns probability to actions of different suppliers (or an action which requires no supplier), then $\delta\left(a_{i}^{j}, \alpha\right)=0$ for any supplier $\lambda\left(a_{i}^{j}\right)$.

\section{Private Contracts}

The game with private contracts, $\Gamma^{\text {pvt }}$, is as follows:

1. Each supplier in $\Lambda=\cup_{i \in I} \Lambda_{i}$ quotes a price for each input that he supplies.

2. Each player $i$ observes the price vector $\mathbf{p}_{i}$ (but not the prices quoted to other players), and players simultaneously choose actions $a_{i} \in A_{i}$.

3. Players receive the net payoffs as defined in (1), and suppliers receive their payoffs. 
If we replace (2) above so that every player observes $\left(\mathbf{p}_{i}\right)_{i \in I}$, the prices quoted to all players, we have a game with public contracts, which we can call $\Gamma^{p u b}$. This is analyzed in the following section.

In either game, a (pure) strategy for a supplier is a price, i.e. a real number. In the game with private contracts, a pure strategy for player $i$ is a function $s_{i}: R^{m_{i}} \rightarrow A_{i}$, where $m_{i}$ is the cardinality of $\Lambda_{i}$.In the game with public contracts, a pure strategy is a map $s_{i}: \times_{j} R^{m_{j}} \rightarrow A_{i}$.

If suppliers choose deterministic prices, a strategy profile in $\Gamma^{p v t}$ consists of a pair $(\hat{\mathbf{p}}, \sigma)$, where $\hat{\mathbf{p}}$ is the vector of prices chosen by all suppliers, and for each player $i, \sigma_{i}: R^{m_{i}} \rightarrow \Delta\left(A_{i}\right)$. Player $i$ 's induced equilibrium (mixed) action is $\sigma_{i}\left(\hat{\mathbf{p}}_{i}\right)$. The (mixed) action profile which is played under this strategy profile is $\sigma(\hat{\mathbf{p}})=\left(\sigma_{i}\left(\hat{\mathbf{p}}_{i}\right)\right)_{i \in I}$, and is called the action outcome of this profile. Now consider randomized prices chosen by suppliers for player $i$, and let $F^{a_{i}^{j}}$ denote the distribution function for prices chosen by supplier $\lambda\left(a_{i}^{j}\right)$. Let $F^{i}$ denote the distribution over price vectors $\mathbf{p}_{i}$ for player $i$. The induced mixed action for player $i$ is $\alpha_{i}=\int \sigma_{i}\left(\mathbf{p}_{i}\right) d F^{i}\left(\mathbf{p}_{i}\right)$, and the action outcome is the vector $\left(\alpha_{i}\right)_{i \in I}$.

The game with private contracts is a game of imperfect information where suppliers have continuum action sets, i.e. a continuum extensive form game (see Simon and Stinchcombe [16]). We focus on Nash equilibria that satisfy sequential rationality, so that each player chooses actions optimally at any profile of supplier prices, $\mathbf{p}_{i}$. This requires that we specify a player's beliefs at out of equilibrium prices. The only beliefs that are directly payoff relevant for player $i$ are beliefs regarding the action profile played by other players in the game. We shall require that a player's beliefs are invariant, i.e. they are the same as his equilibrium beliefs, at any price vector $\mathbf{p}_{i}$. This restriction follows naturally from assumption A3, that no supplier supplies more than one player, and from the fact that different suppliers choose their prices independently. Hence, if $i$ 's supplier deviates, $i$ continues to believe that $j$ 's suppliers have chosen their equilibrium prices, and thereby does not change his beliefs regarding $j$ 's actions. In addition, we rule out equilibria where inactive suppliers (i.e. those who do not make a sale) choose strictly negative prices. Such equilibria are called cautious, and can be ruled out by considerations of trembling hand perfection.

Invariant beliefs are similar to passive beliefs invoked in models with one principal and many agents (see [9], [13]). In our case the rationale is stronger since we have independent "principals" (suppliers); consequently, a deviation 
by one does not indicate a deviation by others. Indeed, if we discretize the price space, and focus on sequential equilibria, a player's beliefs would be invariant at all price profiles. To justify caution, we may restrict attention to equilibria of the continuum game which are limit points of a sequence of trembling hand equilibria of discrete games, as the grid of prices becomes finer. Equilibria with negative prices will not be a limit of such trembling hand perfect equilibria.

The following theorem is the main result of this section.

Theorem $1 \Omega^{\Gamma^{p v t}}=E^{G}$, the equilibrium action outcomes of $\Gamma^{p v t}$ and the equilibria of $G$ coincide. In any profile $\boldsymbol{\alpha}$ that is an equilibrium action outcome of $\Gamma^{\text {pvt }}$, each supplier earns his marginal contribution to player $i$ 's payoff at $\boldsymbol{\alpha}$.

Remark 1 If there is supplier competition, with more than one supplier for an action, the first part of the theorem continues to apply, with the proviso that payoffs in the game $G$ are those net of the the cost of the least cost supplier. The definition of a supplier's marginal contribution must be modified. Specifically, if a profile $\alpha$ is played in an equilibrium of $\Gamma^{\text {pvt }}$, then the payoff to the least cost supplier of action $a_{i}^{j}$ is the minimum of $\delta\left(a_{i}^{j}, \boldsymbol{\alpha}\right)$ and the cost advantage relative to the next best supplier of $a_{i}^{j}$.

Theorem 1 is related to results in the literature on strategic delegation with private contracts (e.g. Katz [11] and Fershtman and Kalai [7]), where principals' payoffs depend upon the actions taken all agents, while an agent cares only the money he receives from the principal. Strategic delegation models are an instance of competing vertical structures. With private contracting, a principal cannot affect the actions taken by other agents. Given transferable utility, each vertical structure takes the actions of the other structure as given, and best responds. In our context, we do not have a unified vertical structure, since several "principals" (suppliers) compete, but the results are similar.

With private contracting in the prisoner's dilemma example of the introduction (see Fig. 1), (D,D) must be played in any equilibrium, and each supplier earns his marginal contribution, $\ell_{i}$. If $\ell_{i}>g_{i}$ the equilibrium involves player $i$ choosing a weakly dominated action, but not if the inequality 
is reversed. ${ }^{1}$

To illustrate mixed strategy equilibria, consider the matching pennies game with an outside option in Fig. 2. Since $b<a / 2$ and $a>0$, the unique equilibrium has player 1 playing $T$ and $B$ each with probability onehalf, while player 2 chooses both her actions with equal probability. Let us assume that player 2 does not have to contract with anyone to play either $L$ or $R$. Player faces a monopoly supplier for action $T$, and another monopoly supplier for action $B$, but can play $O U T$ without contracting with anyone. In any equilibrium of $\Gamma^{p v t}, p_{T}=p_{B}=0$ and the mixing probabilities are as in the equilibrium of $\mathrm{G}$. To see this, let us suppose that we have an equilibrium where $p_{T}>0$, and player 1 randomizes between $T$ and $B$. By choosing $p_{T}-\varepsilon$, the supplier of $T$ ensures that player 1 has a strict incentive to play $T$, and thus strictly positive prices are impossible. In other words, if player $i$ randomizes between any two actions, then the prices paid to these suppliers is zero, since they act as though there are producing perfect substitutes. This maybe called a generalized indifference principle for mixed strategies with private contracts, since the suppliers as well as the player are indifferent between his choice of actions. ${ }^{2}$

\begin{tabular}{|l|l|l|}
\hline & $L$ & $R$ \\
\hline$T$ & $a, 0$ & 0,1 \\
\hline$B$ & 0,1 & $a, 0$ \\
\hline OUT & $b, 0$ & $b, 0$ \\
\hline
\end{tabular}

Fig. 2: Matching Pennies with an Outside Option:2b<a, $a>0$.

Assumption A1, that there are no complementary inputs necessary for taking an action, is essential for our main result. In its absence, coordination failures can ensure that action profiles which are not equilibria of $G$ are equilibrium action outcomes of $\Gamma^{p v t}$ - this is well known from the pricing of complements. More interestingly, this affects mixed equilibria. Let us

\footnotetext{
${ }^{1}$ Simon and Stinchcombe [16] develop equilibrium refinements for infinite normal form games, and argue for limit admissibility - equilibrium strategies should be limits of admissible strategies. In this extensive form example, limit admissibility and sequential rationality preclude existence. However, this equilibrium is a limit of perfect equilibria of the discretized version of the game, where each player has a strict incentive to play $D$.

${ }^{2}$ A companion paper (Bhaskar [3]) explores the implications of generalized indifference in the context of games with imperfect observation played in a contracting environment.
} 
consider the matching pennies game in Fig. 2, and assume that for player 1, no inputs are required for action $T$ and that two inputs are required playing $B$. Let the supplier of the first input for $B$ choose a price equal to $\frac{a}{2}$, while the supplier of the second input randomizes, choosing a price of zero with probability $\frac{1}{2}$, and a price of $\frac{a}{2}$ with probability $\frac{1}{2}$. Player 2 chooses $L$ with probability one-third - different from the mixed equilibrium of $G$. Player 1 chooses $B$ if the total price he has to pay is less than equal to $\frac{a}{2}$, and $T$ otherwise. One supplier makes positive profits at this equilibrium, while the other makes zero profits. That is, at least one of the producers of the complementary goods is indifferent between making a sale or not.

\section{Public Contracts}

We now consider the game with public contracts, $\Gamma^{p u b}$. We focus on cautious subgame perfect equilibria, where supplier prices are non-negative. Let $\Omega^{\Gamma^{p u b}}$ denote the set of equilibrium action outcomes. To ensure existence of equilibrium, we assume that there exists a public signal which is uniformly distributed on $[0,1]$, that is realized after suppliers choose prices, but before players choose actions. Assumption A2 ensures that the maximum price at which any supplier $\lambda\left(a_{i}^{j}\right)$ can make a sale is $\max _{a_{-i}} g_{i}\left(a_{i}^{j}, a_{-i}\right)-\min _{a_{-i}} g_{i}\left(a_{i}^{0}, a_{-i}\right)$. Since supplier strategy sets are effectively compact, the results of Simon and Zame [17] and Harris et. al. [8] ensure that there exists a subgame perfect equilibrium.

\subsection{Pure strategy equilibria}

Consider a game $G$ with a pure strategy Nash equilibrium $\mathbf{a}^{*}=\left(a_{i}^{*}\right)_{i \in I}$. We are interested in the conditions under which $\mathbf{a}^{*}$ is an equilibrium action outcome of $\Gamma^{p u b}$. From the literature on strategic delegation, we know that this will generally not be the case if for some player $i$, the supplier of $a_{i}^{*}$ also controls other actions. For example, if $G$ is the Cournot duopoly game, and only player 1 needs a supplier who controls all his actions (quantity levels), then the unique equilibrium action outcome is the Stackelberg quantity profile. We shall therefore assume:

Assumption A4: For every player $i, \lambda\left(a_{i}^{*}\right)$ does not control any other action. 
We now set out some conditions under which the game $\Gamma^{p u b}$ has an equilibrium with action outcome $\mathbf{a}^{*}$.

Condition C1: Maximality of supplier marginal contributions at $\mathbf{a}^{*}$ : for all $i \in I$, either a supplier is not required for $a_{i}^{*}$ or

$$
a_{-i}^{*} \in \arg \max _{a_{-i} \in A_{-i}}\left\{g_{i}\left(a_{i}^{*}, a_{-i}\right)-\max _{a_{i} \neq a_{i}^{*}} g_{i}\left(a_{i}, a_{-i}\right)\right\} .
$$

Condition C2: Minimality of supplier marginal contributions at $\mathbf{a}^{*}$ : for all $i \in I$, either a supplier is not required for $a_{i}^{*}$ or

$$
a_{-i}^{*} \in \arg \min _{a_{-i} \in A_{-i}}\left\{g_{i}\left(a_{i}^{*}, a_{-i}\right)-\max _{a_{i} \neq a_{i}^{*}} g_{i}\left(a_{i}, a_{-i}\right)\right\} .
$$

Condition C3: $G$ is a symmetric game and $\mathbf{a}^{*}=\left(a^{*}, a^{*}, . ., a^{*}\right)$ is a symmetric pure strategy Nash equilibrium. Let $\tilde{a} \in \arg \max _{a_{i} \neq a^{*}} g_{i}\left(a_{i}, a_{-i}^{*}\right)$ where $a_{-i}^{*}$ denotes the $n-1$ vector where all players other than $i$ choose $a^{*}$. Assume that $\tilde{a} \in \arg \max _{a_{i} \neq a^{*}} g_{i}\left(a_{i}, a_{-i}\right)$ for every profile $a_{-i}$ consisting of elements from the set $\left\{a^{*}, \tilde{a}\right\}$.

An example where the last assumption is satisfied is where $\tilde{a}$ weakly dominates every action other than $a^{*}$.

Condition C4: (multiple equilibria). $G$ has another Nash equilibrium $\left(\alpha_{i}\right)_{i \in I}$ such that for every player $i, a_{i}^{*}$ is not in the support of $\alpha_{i}$.

Theorem 2 Suppose that $G$ has a pure strategy Nash equilibrium $\mathbf{a}^{*}$ where for every player $i$, the supplier of $a_{i}^{*}$ controls no other action. If $G$ satisfies one of the conditions $\mathrm{C1}-\mathrm{C} 4$, there exists an equilibrium of $\Gamma^{\text {pub }}$ with action outcome $\mathbf{a}^{*}$, where each active supplier earns his marginal contribution.

The conditions required for the conclusions of this theorem are rather strong, as can be seen by considering the asymmetric version of the prisoner's dilemma example in the introduction, where $\ell_{1}<g_{1}$ and $\ell_{2}>g_{2}$. We claim that there is no equilibrium where $\mathbf{D}=(D, D)$ is played. To see this, let us suppose that there is such an equilibrium. Clearly, each supplier's price must be less than or equal to $\ell_{i}$, his marginal contribution at $\mathbf{D}$. This implies that $D$ is weakly dominant for 1 in any subgame where his supplier does not deviate. Consequently, 2's supplier can increase his price if it is strictly less than $\ell_{2}$, since in any equilibrium of the resulting subgame, 2 must play $D$ 
with probability one. ${ }^{3}$ However, if $p_{2}=\ell_{2}$, supplier 1 can profitably choose a higher price - if he chooses $p_{1} \in\left(\ell_{1}, g_{1}\right)$, then in any equilibrium of the induced subgame, player 1 must play $D$ with probability one. ${ }^{4}$ Thus supplier 1 can increase his price without reducing the probability of a sale. However, if $p_{1}>\ell_{1}$, $\mathbf{D}$ cannot be played, so that there is no equilibrium with action outcome $\mathbf{D}$.

Since seller 2 can ensure a sale by pricing below $g_{2}$, any equilibrium must involve randomized prices and a non-degenerate (correlated) distribution over action profiles. In one such equilibrium, each seller $i$ randomizes between prices in the set $\left\{g_{i}, \ell_{i}\right\}$, choosing the higher price with probability $\pi_{i}=$ $\frac{\min \left\{\ell_{j}, g_{j}\right\}}{\max \left\{\ell_{j}, g_{j}\right\}}$. Buyers actions, as a function of the price profile, are set out in Fig. 3. It is easy to verify that each supplier is indifferent between the two prices in the support of her mixed strategy, and strictly prefers these to any other prices.

\begin{tabular}{|l|l|l|}
\hline & $g_{2}$ & $\ell_{2}$ \\
\hline$\ell_{1}$ & $D, D$ & $D, D$ \\
\hline$g_{1}$ & $C, D$ & $D, C$ \\
\hline
\end{tabular}

Fig. 3: Actions as a function of supplier prices

This example relies on the marginal contribution of supplier 1 being minimal at $\mathbf{D}$, while that of supplier 2 is maximal. The argument generalizes to many-player games. Let $\mathbf{a}^{*}$ be a pure strategy equilibrium of a game $G$ where for some player $i$, the marginal contribution of his supplier is uniquely minimal at $\mathbf{a}^{*}$ while for some other player $j$, the marginal contribution of his supplier is uniquely maximal at $\mathbf{a}^{*}$ (i.e. the minimizers and maximizers are unique). Consider an equilibrium in $\Gamma^{p u b}$ with action outcome $\mathbf{a}^{*}$ where each supplier prices at his marginal contribution. This makes action $a_{j}^{*}$ weakly dominated for $j$, and $j$ can only play $a_{j}^{*}$ in any subgame where $p_{j}$ equals the marginal contribution if $a_{-j}^{*}$ is played with probability one. Thus supplier $i$ can increase his price slightly, and in any equilibrium of the resulting subgame, his marginal contribution must increase, thereby ensuring that $i$ continues to play $a_{i}^{*}$ for sure.

\footnotetext{
${ }^{3}$ More precisely, if 2 plays $C$ with positive probability, 1 must play $D$ for sure, ensuring that $D$ is strictly better than $C$ as long as the price paid by 2 is strictly less than $\ell_{2}$.

${ }^{4}$ If 1 plays $C$ with positive probablity, then 2 must choose $C$ with probability one (since $D$ is weakly dominated), but this implies that $D$ is strictly better than $C$ for 1 .
} 
This argument however presupposes that the equilibrium where $\mathbf{a}^{*}$ is played has marginal contribution pricing. This is not necessarily the case, since one may be able to construct equilibria where active suppliers price below their marginal contribution, and inactive suppliers may also price above zero. Even if $G$ has a dominant strategy equilibria, with non-zero prices one may have multiple equilibria, allowing players to coordinate punishments on any deviating supplier by switching between equilibria.

We now consider a class of $n$ player prisoner's dilemma games where the argument does generalize. Each player $i \in I$ must choose an action $a_{i} \in\{C, D\}$. The payoff to player $i$ at $\left(a_{i}, a_{-i}\right)$ only depends upon $a_{-i}$ via the number of opponents who choose $C$, i.e. the precise identity of these opponents is irrelevant. $D$ is strictly dominant and $d_{i}^{k}, k \in\{0,1, . ., n-1\}$ denotes the payoff gain to $i$ from playing $D$ when $k$ opponents play $C$ and the remaining $(n-1-k)$ opponents play $D$. We shall assume that for any player, a supplier is required for action $D$ but not for $C$. Let $p_{i}$ denote the price quoted by the supplier for player $i$ for $D$. We are interested in conditions under which the profile $\mathbf{D}=(D, D, \ldots, D)$ is played in equilibrium. A marginal contribution equilibrium at $\mathbf{D}$ is one where $p_{i}=d_{i}^{0} \forall i$.

Define, recursively, the following subsets of the player set $I$.

$I_{1}=\left\{i \in I: d_{i}^{0}<d_{i}^{k} \forall k, 0<k \leq n-1\right\}$. Let $k_{1}$ denote the cardinality of $I_{1}$.

For $h>1, I_{h}=\left\{i \in I: d_{i}^{0}<d_{i}^{k} \forall k, 0<k \leq n-1-k_{h-1}\right\}$. Let $k_{h}$ denote the cardinality of $\cup_{j \leq h} I_{j}$.

This recursive procedure defines a sequence of numbers which converges when $k_{h}=k_{h+1}=k^{*}$.

If $d_{i}^{0}<d_{i}^{k} \forall k>0$, we say that the marginal contribution is uniquely minimal for $i$ at $\mathbf{D}$. and If $d_{j}^{0}>d_{j}^{k} \forall k$, we say that the marginal contribution is uniquely maximal for $j$ at $\mathbf{D}$.

Theorem 3 Let $G$ be a n player prisoner's dilemma game, where for each player, a supplier is required for action $D$ only. i) If $k^{*}=n$, then any equilibrium of $\Gamma^{p u b}$ where $\mathbf{D}$ is played is a marginal contribution equilibrium. ii) If there exist $i, j \in I$ such that at $\mathbf{D}$, the marginal contribution is uniquely minimal for $i$ and uniquely maximal for $i$, there does not exist a marginal contribution equilibrium of $\Gamma^{p u b}$ where $\mathbf{D}$ is played.

The intuition for the marginal contribution pricing result in part (i) is simpler if we assume a discrete price grid that is arbitrarily fine. Assume 
that the price grid does not contain the payoff parameters $d_{i}^{0}$, for every $i,{ }^{5}$ and let $\hat{d}_{i}^{0}$ denote the largest element of the grid that is less than $d_{i}^{0}$. If we have an equilibrium where $\mathbf{D}$ is played, prices cannot exceed $\hat{d}_{i}^{0}$ for any $i$. So any supplier of $i \in I_{1}$ must price at $\hat{d}_{i}^{0}$, since $D$ is strictly is strictly dominant for $i$ at this price. Given this, any supplier of $i \in I_{2}$ must also price at $\hat{d}_{i}^{0}$, and if $k^{*}=n$, this argument iterates for suppliers to any player in $I$. The proof shows that a similar inductive argument also applies in the continuum case, when prices are such that $D$ is iteratively weakly dominant for players.

\begin{tabular}{|l|l|l|l|}
\hline Player & $d^{0}$ & $d^{1}$ & $d^{2}$ \\
\hline 1 & 1 & 2 & 3 \\
\hline 2 & 3 & 2 & 1 \\
\hline 3 & 2 & 3 & 1 \\
\hline
\end{tabular}

\begin{tabular}{|l|l|l|l|}
\hline Player & $d^{0}$ & $d^{1}$ & $d^{2}$ \\
\hline 1 & 1 & 2 & 3 \\
\hline 2 & 3 & 2 & 1 \\
\hline 3 & 3 & 2 & 1 \\
\hline
\end{tabular}

Fig. 4: Two 3-Player Prisoner's Dilemma Games

Two three player examples in Fig. 4 are illustrative. Payoffs are identical across the games for players 1 and 2 , and the marginal contributions at $\mathbf{D}$ are uniquely minimal for player 1 and uniquely maximal for player 2 . In both games, $I_{1}=\{1\}$, so that $d_{j}^{2}$ becomes irrelevant for $j \in\{2,3\}$. In the game on the left, $I_{2}=\{3\}$, and $I_{3}=\{2\}$, so that $k^{*}=3$. This implies that $p_{i}=d_{i}^{0}$ in any equilibrium where $\mathbf{D}$ is played, and by part (i) of the theorem, there cannot be such an equilibrium. In the game on the right, the iterative process stops after one step, since $I_{2}=\emptyset$. An equilibrium where prices are below their marginal contributions is as follows: $p_{1}=1, p_{2}=p_{3}=2$, and $\mathbf{D}$ is played a this profile. If either supplier 2 or supplier 3 deviate and choose a higher price, $(D, C, C)$ is played. If supplier 1 deviates to a higher price, then $(C, D, D)$ is played. Since the price are below the marginal contribution for players 2 and 3 , it is optimal to continue playing $D$ even when player 1 switches to $C$. Note that this equilibrium construction relies on continuum supplier strategy sets, since a price of 2 allows players 2 (and player 3 ) to be indifferent between $C$ and $D$ when exactly one of his opponents plays $C .{ }^{6}$

\footnotetext{
${ }^{5}$ This would be the case generically, e.g. if payoffs were irrational while any price grid was restricted to the rationals.

${ }^{6}$ This raises an interesting question, on the nature of equilibria that are robust to arbitrary discretizations, and the appropriate notions of genericity in this context. We leave this for future work.
} 


\subsection{Mixed strategy equilibria}

Suppose that the game $G$ has a unique mixed equilibrium. We now show, via an example, that under public contracting, complex behavior emerges. Competing suppliers for a player act partly as though they produce perfect complements. Consider the matching pennies game of Fig. 2, where for player 1, there is a monopoly supplier for action $T$ and another supplier for action $B$. Player 2 requires no supplier for either action. Let $p_{j}, j \in$ $\{T, B\}$ denote the prices to be paid for playing $j$. If $\left|p_{T}-p_{B}\right| \leq a$, and if we exclude OUT momentarily from consideration, the (restricted) subgame has a completely mixed equilibrium where player 1 plays $T$ and $B$ each with probability one-half and player 2 plays $L$ with probability $\pi=\frac{1}{2}+\frac{p_{T}-p_{B}}{2 a}$. The expected payoff to player 1 in this equilibrium equals $\frac{a-\left(p_{T}+p_{B}\right)}{2}$, and this must be greater than $b$ or else OUT will be better. The probability with which $T$ is played equals one-half - so as to keep player 2 indifferent between his two actions. This is therefore independent of the value of $p_{T}$ or $p_{B}$, so long as $\left|p_{T}-p_{B}\right| \leq a$. Hence supplier $T$ chooses $p_{T}$ to maximize $\frac{p_{T}}{2}$ subject to the constraint $\frac{a-\left(p_{T}+p_{B}\right)}{2} \geq b$, and supplier $B$ chooses $p_{B}$ to maximize $\frac{p_{B}}{2}$, also subject to the same constraint. Candidate equilibria are non-negative values of $p_{T}, p_{B}$ such that

$$
p_{T}+p_{B}=a-2 b .
$$

Since each supplier can sell for sure by undercutting his rival's price by $a$, this implies the constraint:

$$
p_{j} \leq a-\frac{2 b}{3}, j \in\{T, B\}
$$

Now if $b>-\frac{3 a}{2}$, conditions (2) and (3) are consistent, and we have a continuum of equilibria. In particular, if $p_{T}=p_{B}$, player 1 chooses $T$ and $B$ each with probability one-half, so that the action outcome corresponds to the mixed equilibrium of $G$. When the outside option bites, the suppliers of the two actions act as though they are producing complementary goods that combine to form a single composite good, that must compete with the outside option. This gives rise to a continuum of pricing equilibria and equilibrium distributions over action profiles.

If $b<-\frac{3 a}{2}$ we cannot have an equilibrium with deterministic prices since conditions (2) and (3) are inconsistent. If supplier $k$ prices low, then it is 
better to price high, but if he prices high, then $j$ would prefer to undercut and take the entire market, and so a deterministic price equilibrium fails to exist. Let us assume that $b$ is sufficiently low that it becomes irrelevant. We construct a symmetric mixed equilibrium where the price chosen by each supplier, $x$, has continuous distribution $F$. The payoff to a supplier from choosing $x$ is given by

$$
\begin{gathered}
U(x, F)=x[1-F(x+a)]+\frac{x}{2}[F(x+a)-F(x-a)] . \\
\frac{\partial U}{\partial x}=2-[F(x+a)+F(x-a)]-x\left[F^{\prime}(x+a)+F^{\prime}(x-a)\right]=0, x \in[\underline{x}, \bar{x}] .
\end{gathered}
$$

The solution to this differential equation (5) is given by the following distribution, on support $[a \sqrt{2}, a(\sqrt{2}+2)]$ :

$$
F(x)=\left\{\begin{array}{c}
1-\frac{a(1+\sqrt{2})}{x+a}, x \in[a \sqrt{2}, a(1+\sqrt{2})] \\
2-\frac{a(1+\sqrt{2})}{x-a}, x \in[a(1+\sqrt{2}), a(2+\sqrt{2})] .
\end{array}\right.
$$

The payoff of a supplier in this equilibrium equals $\frac{a(1+\sqrt{2})}{2}$.In this equilibrium the two suppliers act as though the good they produce are partly complements and partly substitutes. If my price is close to that of my competitor, then my payoff is increasing in my own price, since demand is inelastic. However, if two prices are sufficiently far apart, then the lower priced supplier takes the market for sure. The action outcome is a correlated distribution over action profiles. If the difference between supplier prices exceeds $a$, a pure profile is played (i.e. either $(B, L)$ or $(T, R))$. When the difference is less than $a, T$ and $B$ are played with probability one-half, while the probability of $L$ depends upon the prices.

To conclude, this paper has analyzed games where players have to contract with other suppliers in order to take actions. As in the literature on principal-multi agent games, we find that it is important whether contracts are public (i.e. observed by all the players in the game) or private (observed only by contracting parties). We also find that there is a major difference between mixed equilibrium in the game, where players randomize between their actions, and pure equilibria. 


\section{Appendix}

Proof of Theorem 1: Let $\boldsymbol{\alpha}$ be an equilibrium of $G$. We first construct an equilibrium that induces the action outcome $\boldsymbol{\alpha}$. If $\alpha_{i}$ assigns probability only to actions which belong to a single supplier, let the price of any such action $\left(a_{i}^{k}\right)$ equal $\hat{p}_{i}^{k}=\delta\left(a_{i}^{k}, \alpha\right)$, and let this be the price of every action supplied by $\lambda\left(a_{i}^{k}\right)$. Let $\hat{p}_{i}^{j}=0$ for all inactive suppliers $\lambda\left(a_{i}^{j}\right)$. If $\alpha_{i}$ assigns positive probability to the actions supplied by more than one supplier or to an action that does not require a supplier, prices are zero for all suppliers. Player $i$ chooses $\alpha_{i}$ in response to the equilibrium price vector $\hat{\mathbf{p}}_{i}$. He chooses any best response after any other prices; in particular, if supplier $\lambda\left(a_{i}^{j}\right)$ chooses a higher price, player $i$ does not buy from him with probability one. The beliefs of player $i$ equal $\alpha_{-i}$ at every $\mathbf{p}_{i}$, and satisfy the invariant beliefs criterion. From the definition of $\hat{\mathbf{p}}_{i}$ it is optimal for $i$ to choose $\alpha_{i}$. Furthermore, since prices are such that $i$ is indifferent from buying or not buying from any active supplier, it is optimal to not buy if any supplier increases his price.

We now show that any equilibrium of $\Gamma^{p v t}$ has an action outcome that belongs to $E^{G}$. Fix an equilibrium of $\Gamma^{p v t}$, and note that from the point of view of player $i$ and his suppliers, the strategies of the other suppliers and the other players induce a mixed action profile, $\alpha_{-i}$. By our assumption of invariant beliefs, player $i$ 's beliefs about the profile played by other players do not change with the prices charged by $i$ 's suppliers. So our results follow from standard results on monopoly pricing and on Bertrand competition between asymmetric sellers and a single buyer. Let $a_{i}^{j} \in \arg \max _{a_{i}} g_{i}\left(a_{i}, \alpha_{-i}\right)$, and let this action be supplied by $\lambda\left(a_{i}^{j}\right)$. If $\lambda\left(a_{i}^{j}\right)$ also supplies an action that yields a strictly lower payoff, then this cannot be purchased in equilibrium - at any price at which this action is sold, there exists a price for $a_{i}^{j}$ that makes both supplier and player strictly better off. Let $a_{i}^{k} \in \arg \max _{a_{i}: \lambda\left(a_{i}\right) \neq \lambda\left(a_{i}^{j}\right)} g_{i}\left(a_{i}, \alpha_{-i}\right)$ be the next best action that is not supplied by $\lambda\left(a_{i}^{j}\right)$. Consider first the case where $g_{i}\left(a_{i}^{j}, \alpha_{-i}\right)>g_{i}\left(a_{i}^{k}, \alpha_{-i}\right)$. In this case, we show that actions belonging to $\lambda\left(a_{i}^{j}\right)$ must be played with probability one, and supplier $\lambda\left(a_{i}^{j}\right)$ must earn a payoff $\delta\left(a_{i}^{j}, \alpha_{-i}\right)$ in any cautious equilibrium. By choosing a price $\delta\left(a_{i}^{j}, \alpha_{-i}\right)-\varepsilon, \lambda\left(a_{i}^{j}\right)$ can ensure a sale with probability one, since the prices of other suppliers are non-negative, and thus his payoff must be no less than $\delta_{i}\left(a_{i}^{j}, \alpha_{-i}\right)$. If his payoff is strictly greater than $\delta\left(a_{i}^{j}, \alpha_{-i}\right)$, then the support of $\lambda\left(a_{i}^{j}\right)$ 's mixed strategy must consist of prices strictly greater than $\delta\left(a_{i}^{j}, \alpha_{-i}\right)$ and $\lambda\left(a_{i}^{k}\right)$ can also earn positive profits. Thus in any mixed strat- 
egy equilibrium suppliers $\lambda\left(a_{i}^{j}\right)$ and $\lambda\left(a_{i}^{k}\right)$ must both earn positive profits. By assumption A2, the prices of the two sellers must also be bounded above. We now show that at least one seller's mixed strategy has in its support a price that earns zero profits, contradicting our earlier result that each seller earns positive profits. Let $x\left(\lambda\left(a_{i}^{j}\right)\right)$ (resp. $x\left(\lambda\left(a_{i}^{k}\right)\right)$ ) denote the supremum of prices that lie in the support of $\lambda\left(a_{i}^{j}\right)$ 's (resp. $x\left(\lambda\left(a_{i}^{k}\right)\right)$ mixed strategy. If $x\left(\lambda\left(a_{i}^{j}\right)\right)>x\left(\lambda\left(a_{i}^{k}\right)\right)+\delta\left(a_{i}^{j}, \alpha_{-i}\right)$, then $\lambda\left(a_{i}^{j}\right)$ chooses a price which makes zero profits, while if the inequality is reversed, this is the case for $\lambda\left(a_{i}^{k}\right)$. If both expressions are equal, then both sellers must choose a price which makes zero profits, unless each player's mixed strategy has an atom at the supremum. But in this case, a player can do strictly better by choosing a price $\varepsilon$ below the supremum, where $\varepsilon$ is sufficiently small. We conclude therefore that if actions supplied by $\lambda\left(a_{i}^{j}\right)$ are the only maximizers $g_{i}\left(a_{i}, \alpha_{-i}\right)$, then only these actions must be played with positive probability, and $\lambda\left(a_{i}^{j}\right)$ earns his marginal contribution. Similar arguments establish that if $\arg \max _{a_{i}} g_{i}\left(a_{i}, \alpha_{-i}\right)$ has several elements that are supplied by distinct suppliers (or if one of them does not require a supplier), then the player's mixed strategy can only assign probability to one of these, and the prices must equal zero.

We have therefore shown that in any equilibrium of $\Gamma^{p v t}$, each player must assign positive probability only to actions that maximize his gross payoffs, given his equilibrium beliefs regarding the actions of other players. Thus the action outcome of must be an element of $E^{G}$, and supplier payoffs are as in the statement of the theorem.

Proof of Theorem 2: We construct equilibria, where for each $i$, any active supplier $\lambda\left(a_{i}^{*}\right)$ chooses price equal to $\delta\left(a_{i}^{*}, a^{*}\right)$, and every other supplier chooses a price of zero. In the resulting subgame, it is clearly an equilibrium for each player to choose $a_{i}^{*}$. It remains to specify player behavior after a deviation by any one seller, $\lambda\left(a_{i}^{*}\right)$, to a higher price, so that such a deviation is not profitable, and the details of this depend on which of the conditions C1-C4 holds.

If $\mathrm{C} 1$ holds, no supplier can possibly earn any more, since the payoff he gets is maximum that the player would be willing to pay, over all profiles of his opponents.

Suppose C2 holds. If active supplier $\lambda\left(a_{i}^{*}\right)$ deviates by choosing a higher price, then all players $j \neq i$ continue to choose action $a_{j}^{*}$, while player $i$ chooses the action

$$
a_{i}^{\prime} \in \arg \max _{a_{i} \neq a_{i}^{*}} g_{i}\left(a_{i}, a_{-i}^{*}\right)
$$


This response by player $i$ makes a price increase for the supplier unprofitable. It remains to verify that the continuation play constitutes a Nash equilibrium in the subgame. For player $i$, choosing $a_{i}^{\prime}$ rather than $a_{i}^{*}$ is clearly preferable since the price chosen by supplier $\lambda\left(a_{i}^{*}\right)$ is greater than $\delta\left(a_{i}^{*}, \mathbf{a}^{*}\right)$. For every player $j$ different from $i$, continuing to play $a_{j}^{*}$ is a best response since the marginal contribution at any profile $\left(a_{j}^{*}, a_{-j}\right)$ is greater than the price $\delta\left(a_{i}^{*}, \mathbf{a}^{*}\right)$.

Suppose C3 holds. Let $d^{k}, k=0,1, . ., n-1$ denote the payoff gain to a player from choosing $a^{*}$ given that $i$ opponents are choosing $\tilde{a}$ and while $n-1-k$ opponents choose $a^{*}$. We focus on an equilibrium where for each player, his supplier of $a^{*}$ prices at $d^{0}$, and all other suppliers price at zero. At the equilibrium price profile, each player chooses $a^{*}$ - this is clearly optimal from the definition of $d^{0}$. Players ignore deviant price increases by inactive suppliers and continue to play $a^{*}$. It remains to specify player strategies so that no active supplier can profitably deviate. Suppose that the supplier of $a^{*}$ for player $i$ deviates to a higher price:

a) If $d^{1} \geq d^{0}$, player $i$ plays $\tilde{a}$ while all other players continue playing $a^{*}$.

b) If $d^{n-1} \leq d^{0}$, all players switch to playing $\tilde{a}$.

c) If $d^{1}<d^{0}<d^{n-1}$, there exists an integer $k$, such that $1 \leq k<n-1$ and $g^{k-1} \leq g^{0}$ and $g^{k} \geq g^{0}$. Let $k$ players (including $i$ ) choose $\tilde{a}$, while the remainder choose $a^{*}$.

Suppose C4 holds. Upon a price increase by any supplier $\lambda\left(a_{i}^{*}\right)$, the players play $\alpha$, so that the deviant supplier gets zero. Since $\alpha$ is an equilibrium of $G$, this is an equilibrium in this subgame since only the payoff to action $a_{i}^{*}$, which is not in the support of $\alpha_{i}$, has been reduced.

Proof of Theorem 3: i) For any player $i \in I_{1}, D$ is strictly dominant as long as $p_{i}<d_{i}^{0}$, and hence no such price can be optimal for the supplier. Note that if $p_{i}=d_{i}^{0}, D$ is weakly dominant for $i \in I_{1}$, and $C$ can only be played by $i$ if every other player chooses $D$ with probability one.

Consider $i \in I_{2}$, and suppose that $p_{i}<d_{i}^{0}$. We claim that this cannot be optimal since supplier $i$ can increase his price to $p_{i}^{\prime}<d_{i}^{0}$ and $i$ must continue to play $D$ with probability one. Suppose the contrary, that $i$ plays $C$ with positive probability. This implies that every player in $I_{1}$ must choose $D$, implying that $i$ 's payoff gain from $D$ is a convex combination of values in the set $\left\{d_{i}^{k}: 0 \leq k \leq n-1-k_{1}\right\} \geq d_{i}^{0}>p_{i}^{\prime}$, and hence $i$ must play $D$ with probability one.

Consider $i \in I_{h}$, and suppose $p_{j} \leq d_{j}^{0} \forall j \in I$, and $p_{i}<d_{i}^{0}$. We claim 
that this cannot be optimal since supplier $i$ can increase his price to $p_{i}^{\prime}<d_{i}^{0}$ and $i$ must continue to play $D$ with probability one. Suppose the contrary, that $i$ plays $C$ with positive probability. This implies that every player in $I_{1}$ must choose $D$, which implies that every player in $I_{2}$ must choose $D$, and iteratively, every player in the set $I_{k}, k \leq h-1$ must choose $D$. This implies that $i$ 's payoff gain from $D$ is a convex combination of values in the set $\left\{d_{i}^{k}: 0 \leq k \leq n-1-k_{h-1}\right\} \geq d_{i}^{0}>p_{i}^{\prime}$, and hence $i$ cannot play $C$ with positive probability. This proves that if $k^{*}=n$, then $p_{i}=d_{i}^{0} \forall i \in I$.

ii) Suppose that there exists a marginal contribution equilibrium where $\mathbf{D}$ is played. Let supplier $i$ increase price to $p_{i} \in\left(d_{i}^{0}, \min _{k \neq 0} d_{i}^{k}\right)$. We claim that $i$ must continue to play $D$ with probability one. To see this, suppose otherwise; this implies that $D$ is strictly worse than $C$ for $j$, since his gross payoff gain from $D$ is a convex combination of elements from the set $\left\{d_{j}^{k}: k>0\right\}$, which is strictly less than the price $d_{j}^{0}$. Since $j$ cannot play $D$ with positive probability, this implies that the payoff gain for $i$ is a convex combination of elements from the set $\left\{d_{i}^{k}: k>0\right\} \geq \min _{k \neq 0} d_{i}^{k}$. Thus $i$ strictly prefers $D$ to $C$, and we have a contradiction.

\section{References}

[1] M. Baye and J. Morgan (1999). A Folk theorem for One Shot Bertrand Games, Economics Letters, 65, 59-65.

[2] B.D. Bernheim and M. Whinston (1987). Menu Auctions, Resource Allocation and Economic Welfare, Quarterly Journal of Economics 104, $1,1-31$.

[3] V. Bhaskar (2007). Commitment and Observability in a Contracting Environment, mimeo.

[4] B. Caillaud, B. Jullien and P. Picard (1995). Competing Vertical Structures: Precommitment and Renegotiation, Econometrica 63, 621-646.

[5] C. Fershtman and K.L. Judd (1987). Incentive Equilibrium in Oligopoly, American Economic Review 77, 927-940.

[6] C. Fershtman, K.L. Judd and E. Kalai (1991). Observable Contracts: Strategic Delegation and Cooperation, International Economic Review $32,551-559$. 
[7] C. Fershtman and E. Kalai (1997). Unobserved Delegation, International Economic Review 38, 763-774.

[8] C. Harris, P. Reny and A. Robson (1995). The Existence of Subgame Perfect Equilibrium in Continuous Games with Almost Perfect Information: A Case for Public Randomization, Econometrica 63,507-545.

[9] O. Hart and J. Tirole (1990). Vertical Integration and Market Foreclosure, Brookings Papers on Economic Activity, 205-276.

[10] M.O. Jackson and S. Wilkie (2005). Endogenous Games and Mechanisms: Side Payments Among Players, Review of Economic Studies 72, 543-566.

[11] M. Katz (1991) Game-Playing Agents: Unobservable Contracts as Precommitments, Rand Journal of Economics 22, 307-328.

[12] L. Kockesen and E. A. Ok (2004). Strategic Delegation by Unobservable Incentive Contracts, Review of Economic Studies 71, 397-424.

[13] R.P. McAfee and M. Schwartz (1994). Opportunism in Multilateral Vertical Contracting: Non-discrimination, Exclusivity and Uniformity, American Economic Review 84, 210-230.

[14] A. Prat and A. Rustichini (2003). Games Played Through Agents, Econometrica 71, 989-1026.

[15] I. Segal (1999). Contracting with Externalities, Quarterly Journal of Economics 104, 337-388.

[16] L. K. Simon and M.B. Stinchcombe (1995). Equilibrium Refinement for Infinite Normal Form Games, Econometrica 63, 1421-1445.

[17] L. K. Simon and W. Zame (1988). Discontinuous Games and Endogenous Sharing Rules, Econometrica 58, 861-872.

[18] S. Sklivas (1987). The Strategic Choice of Managerial Incentives, Rand Journal of Economics 18, 452-458.

[19] J. Vickers (1985).Delegation and the Theory of the Firm, Economic Journal 95, 138-147. 